\title{
The Willed Body Donor Interview Project: Medical Student and Donor Expectations
}

\author{
Michael Bohl, ${ }^{1}$ Alexis Holman, ${ }^{2}$ Dean A. Mueller, ${ }^{2}$ Larry D. Gruppen, ${ }^{3}$ Sabine Hildebrandt ${ }^{2 *}$ \\ ${ }^{1}$ University of Michigan Medical School, Ann Arbor, Michigan \\ ${ }^{2}$ Department of Medical Education, Division of Anatomical Sciences, University of Michigan Medical School, \\ Ann Arbor, Michigan \\ ${ }^{3}$ Department of Medical Education, University of Michigan Medical School, Ann Arbor, Michigan
}

The Anatomical Donations Program at the University of Michigan Medical School (UMMS) has begun a multiphase project wherein interviews of donors will be recorded and later shown to medical students who participate in the anatomical dissection course. The first phase of this project included surveys of both current UMMS medical students and donors concerning their perceptions of such a program. A five-question survey administered via Qualtrics software was electronically mailed to all current medical students at UMMS, and a survey was mailed to registered and potential donors requesting information from the UMMS on anatomical donations. A total of 224 medical student responses (response rate 33\%) and 54 donor responses (response rate 27\%) were received. Seventy-four percent of students and $81 \%$ of donors reported they would participate in this program if it existed. Students and donors supported the implementation of this program for varying reasons, though many felt strongly they would not want to participate in a donor interview program. These qualitative results support those of previous studies that show a majority of students desire a closer personal relationship with the donor, and these are the first results to be reported on donor perceptions of a donor interview program. Although many students and donors are in favor of instituting this program, others feel strongly that such an experience could be traumatic. The causes of these differing reactions need to be further explored, and the opinions of those who object to this study will be respected by maintaining voluntary participation in future phases of this study. Anat Sci Educ 6: 90-100. @ 2012 American Association of Anatomists.

Key words: willed body program; anatomical bequest program; body donors; gross anatomy education; anatomical dissection; perception of anatomical dissection course; empathy; clinical detachment

\section{INTRODUCTION}

While the anonymization of willed body donors is still standard in most Western medical schools, numerous studies have shown that students desire and may benefit from a more per-

${ }^{*}$ Correspondence to: Dr. Sabine Hildebrandt, Department of Medical Education, Division of Anatomical Sciences, University of Michigan Medical School, 3767 Medical Science Building II, Catherine Street, Ann Arbor, MI 48109-0608, USA. E-mail: shilde@umich.edu

Received 10 July 2012; Revised 20 August 2012; Accepted 20 September 2012.

Published online 26 October 2012 in Wiley Online Library (wileyonlinelibrary.com). DOI 10.1002/ase.1326

(C) 2012 American Association of Anatomists sonal relationship with the donor. Coulehan et al. (1995) reported that medical students were curious about the personal history of their donor and felt they needed a student-donor relationship during the course of dissection. Weeks et al. (1995) provided medical students not just with the donors' medical history but also their names. The results of their study led them to suggest that supplying as much personal and medical history of donors as possible to interested students would reinforce respect and compassion in students for donors, citing their own experience and that of others (Penney, 1985; Wear, 1989; Druce and Johnson, 1994). Talarico and Prather considered the frequent practice of medical students' renaming of an anonymous donor as disrespectful and diminishing the donor's dignity (Talarico and Prather, 2007). As a consequence they developed a program in which medical students contact the donors' families and learn their 
names to promote a respectful relationship (Quilligan, 2010). At the University of Oklahoma medical students actually meet the family members of the donor they will be dissecting at an annual luncheon. Students who participated in this program were found to worry less about the suffering of the donor, believe that dissection was not an invasion of the donor's privacy, and viewed the donor more as a person throughout the dissection course as compared with those students who did not meet with their donor's family members (Vannatta and Crow, 2007; Crow et al., 2012). A recent study by Bohl et al. (2011) revealed that a large number of medical students at the University of Michigan desire a closer personal relationship with the donor than the current anonymized approach allows.

In a video project entitled Donated to Science at the University of Otago, New Zealand (Trotman, 2009), medical students were shown a video interview of their assigned donor that was made before that person's death. The impact on the students was profound, and the insight they reportedly gained likely invaluable to their formation as caring physicians. The effect on the donors of video interviewing also appeared significant, though this was not specifically addressed in the project. Some of the participating donors expressed gratitude for the chance to pass along something of their history and personality with their remains, and some even gave their dissectors permission to "take me apart bit by bit," not wanting any of their gifted remains to go to waste (Trotman, 2009).

The current study is based on the hypothesis that a program wherein potential donors video-record an interview for their student dissector highlighting aspects of the donor's personal life, reasons for donating, and family members' memories of the donor, may facilitate a closer personal relationship between medical students and their donors and so lead to a more satisfying experience of the course of human dissection. Encouraging a closer relationship between student and donor might benefit the donor as much as the student, as it could give the donor the opportunity to share with the student their reasons for donating and their expectation to be respected as a person by the student dissecting them. Such a program might also advance the integration of end-of-life care issues into the anatomy curriculum, which could pose a potential benefit both to students just beginning to acknowledge and express their feelings towards death, and future donors whose end-of-life care may one day be managed by those same students (Bertman and Marks, 1985; Rizzolo, 2002). Before initiating a donor interview program further questions of student and donor attitudes have to be explored, as there is no published information on these subjects available at this time. Some students may find the experience of dissection already too distressing and may desire not to have a more personal relationship with their donor when offered. Likewise, certain donors may wish to remain anonymous and pass on as little personal information with their remains as possible. To better understand the range and respective prevalence of student and donor opinions regarding the proposed program, surveys were sent to students at UMMS as well as people who contacted the University of Michigan Anatomical Donations Program and requested information on anatomical donation. The results of these surveys will inform future decisions regarding the implementation of a donor interview program at the University of Michigan, as well as contribute to a growing body of knowledge regarding the relationship between body donors and medical students.

\section{METHODS}

The study was approved by the Institutional Review Board (IRB) of the University of Michigan under IRB protocol \#HUM00060553.

\section{Survey of Medical Students}

A survey of medical students was written with the intention of clarifying whether students believe that watching a donor interview would be helpful or harmful to them during the anatomical dissection course, and to gauge how strongly some students support or oppose the creation of a donor interview program. From 20 February 2012 to 20 March 2012 all currently enrolled University of Michigan medical students ( $N=680)$ were invited via e-mail to participate in a onetime voluntary and confidential survey. The survey invitation included a primer that gave a short description of the proposed donor interview project and a link to the survey (see Appendix A). The survey itself contained a more detailed description of the proposed donor interview project.

The survey contained five questions. It was created and the data analyzed using Qualtrics survey software, version 30888, (Qualtrics Labs Inc., Provo, UT). The full text of the survey is found in Appendix B. All questions except question 4 were scored on a 3 -point scale $(1=$ no, $2=$ I don't know, 3 = yes). Question 4 was scored on a 5 -point scale $(1=$ not at all valuable, $5=$ extremely valuable). Space was left at the end of the survey for students to comment on why they do or do not support a donor interview program, as well as any other thoughts they might have.

\section{Survey of Donors/ Potential Donors}

The Anatomical Donations Program of the University of Michigan receives 15 to 30 phone calls and letters a week from potential donors, registered donors, and family members of donors with questions about body donation. For this project, potential donors were mailed a package of information about the donation procedure and the Donations Program itself. Registered donors received additional information either verbally during the phone call or, if requested, were sent additional material by mail.

Beginning on 15 March 2012, the packages of information mailed to donors and potential donors included a primer on the donor interview project and instructions on how to access a survey regarding the donor's opinions of this project (see Appendix C). A request was made to survey respondents to complete the survey before calling the Anatomical Donations Program with questions regarding the donor interview project in order to avoid affecting the survey data through the sharing of author biases with respondents.

The survey of donors was designed to include a short introduction of the concept of using videotaped interviews with donors as a means to improve the dissection course experience for medical students, followed by nine questions asking donors about their opinions on the subject matter. Respondents were asked to answer questions on a 3-point scale $(1=$ no, $2=$ I don't know, $3=$ yes) or a 5 -point scale $(1=$ very negative, $5=$ very positive). Space was provided for respondents to comment on any worries or reservations they had concerning their gift, their feelings about conducting an interview before death, and any other comments they wanted to make. Potential donors were made aware that their participation in this survey was anonymous and that all 


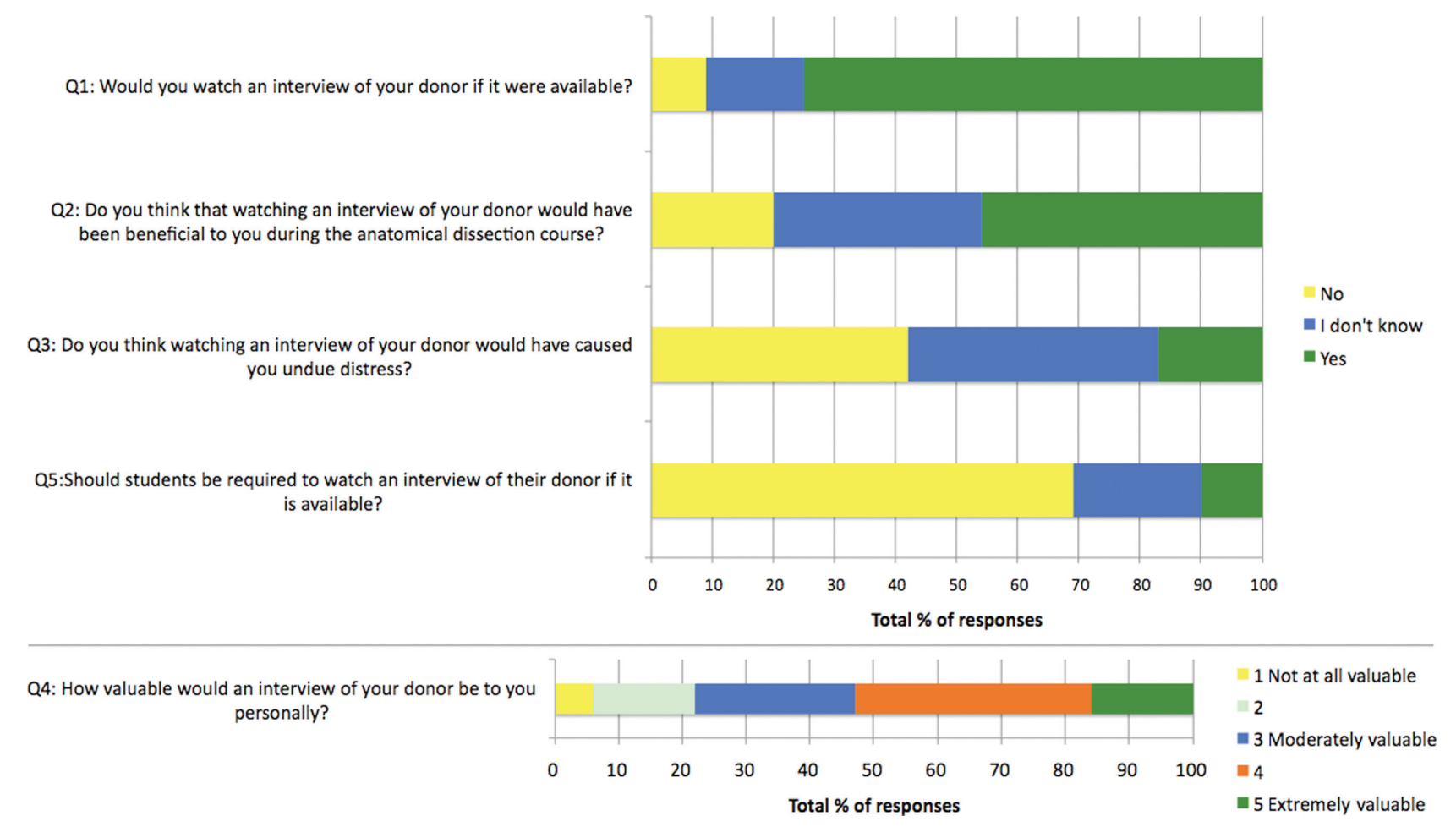

\section{Figure 1.}

Student survey results.

of their responses would remain confidential. The full text of the survey can be found in Appendix D.

Self-addressed and stamped envelopes were included with the hard copies of the survey. Hard copies of the survey that were received back from respondents were separated from any identifying information included in the return envelope by the administrators of the Anatomical Donations Program. De-identified surveys were then collected by the authors and the survey data were entered into Qualtrics survey software. Collection of surveys ended on 30 July 2012 for an open survey period of 4 months.

\section{Evaluation of Student and Donor Survey Data}

The results of the survey questions for donors and students were registered as percentage-points for each of the qualitative categories of the individual question. Comments were read by the authors and grouped into major themes based on content. Some comments addressed multiple themes and so were counted multiple times in the tallying of the various themes.

\section{RESULTS}

\section{Survey of Medical Students}

The survey was e-mailed to approximately 680 medical students at the University of Michigan, and 224 responses were received for an approximate response rate of $33 \%$. This number was considered sufficient for a pilot study concerning a qualitative question. Figure 1 summarizes the distribution of student responses for each question. A large majority of student respondents $(74 \%)$ reported that they would watch an interview of the donor they dissected if it were made available to them, with $16 \%$ reporting they did not know, and $9 \%$ reporting they would not watch the interview. A smaller majority of students $(46 \%)$ reported that they thought watching an interview of their donor would have been helpful to them during the anatomical dissection course, with $34 \%$ reporting they were unsure, and $20 \%$ reporting that watching the interview would not have been helpful to them. Equal majorities of student respondents reported that watching an interview of their donor would not have caused them undue distress $(42 \%)$ or were unsure if it would have caused them undue distress $(41 \%)$, while $17 \%$ reported that watching the interview would have caused them undue distress. When asked to score on a 5 -point scale how personally valuable a donor interview would have been to them, $16 \%$ of students said $5 / 5$ (extremely valuable), $37 \%$ said $4 / 5,25 \%$ said $3 / 5$ (moderately valuable), $16 \%$ said $2 / 5$, and $7 \%$ said $1 / 5$ (not at all valuable). In other words, $78 \%$ of students reported that an interview of their donor would have been at least moderately valuable to them personally. When asked whether they thought that students should be required to watch a donor interview if available in order to honor that person being dissected, $69 \%$ of students said no, $21 \%$ were unsure, and $10 \%$ said yes.

One hundred forty-nine respondents left comments at the end of the survey. Numerous themes were recurrent among these comments. One hundred and eleven respondents sup- 


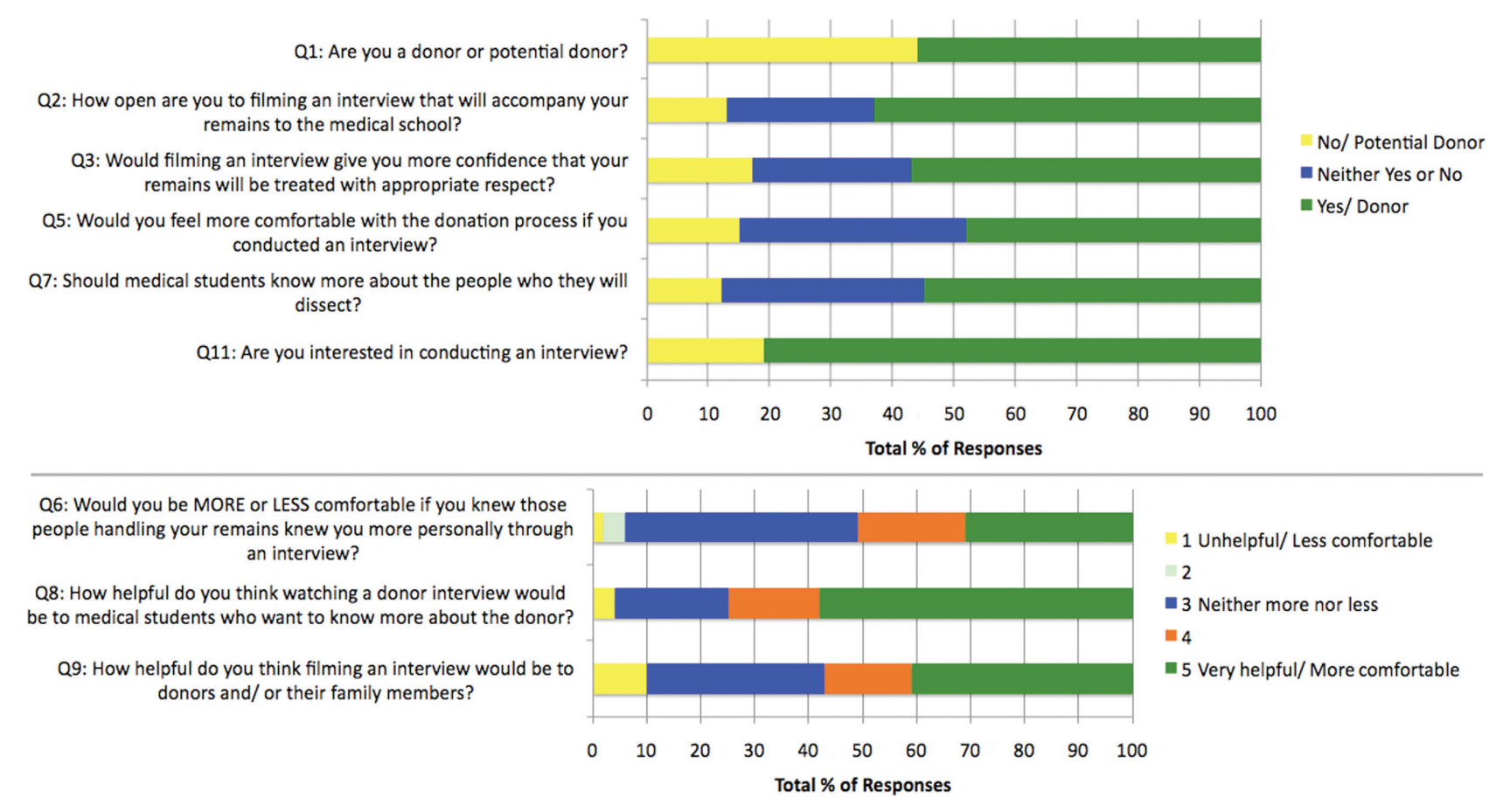

Figure 2.

Donor survey results.

ported the increased empathy and respect for donor personhood that could theoretically result from a donor interview program. On the other hand, 77 comments mentioned a problematic loss of clinical detachment that might result from watching a donor interview. Forty-seven respondents discussed the proposal in terms of its effect on the balance of empathy and clinical detachment that students often struggle to find in the anatomy laboratory. Thirty-four students voiced opposition to making this a mandatory experience for students, whereas 10 students expressed conviction that even though watching an interview would be difficult, students would ultimately be better, more empathetic physicians for having watched the interview. Seventeen students expressed a preference to watch the interview upon the completion of the dissection course. Five students reported that a donor interview would enhance their anatomy education and encourage them to spend more time studying anatomy by increasing the clinical relevance of anatomical dissection. Four students commented that this program could be valuable to donors. Four students cited the positive effect this program would have on student respect for donor remains, two of which commented on having seen behavior in the anatomy laboratory they thought was disrespectful. Finally, three students wrote that they are donors or are considering becoming donors and that they would want their own dissectors to watch a videotaped interview of themselves during the time their remains were in the anatomy laboratory.

\section{Survey of Donors}

Two hundred surveys were mailed to potential donors and registered donors between 15 March 2012 and 30 July 2012.
To date, 54 responses have been received. Although a very low response rate $(27 \%)$, this number was consistent with the return rate of fully completed donor registrations typically seen from this population of people requesting information on anatomical donations. This means that almost all donors who registered with the donation program also returned a survey form. The response rate was also considered sufficient for a pilot study intended to provide a first insight into the range and approximate prevalence of donor opinions regarding the interview project described above. Figure 2 summarizes the distribution of donor responses for each question. Twenty-nine $(56 \%)$ respondents were donors and $23(44 \%)$ were potential donors. When asked how open they were to filming an interview, 34 respondents (63\%) said they were very open/would love to do an interview, 13 (24\%) were ambiguous, and $7(13 \%)$ were very closed to the idea. Thirty respondents $(57 \%)$ reported that filming an interview would have given them more confidence that their remains would be treated with appropriate respect by medical students and faculty, while $14(26 \%)$ were ambiguous, and 9 $(17 \%)$ did not feel this way. A small majority of respondents reported a greater predicted confidence with the donation process if they knew an interview would accompany their remains (25 respondents, 48\%), 19 (37\%) were ambiguous to this notion, whereas $8(15 \%)$ reported no predicted increase in confidence with the donation process. When asked to score on a 5-point scale the effect of an interview on their comfort level with donation, 16 (31\%) scored 5/5 (much more comfortable), $10(20 \%)$ scored 4/5, 22 (43\%) scored 3/ 5 (neither more nor less comfortable), 2 (4\%) scored $2 / 5$, and $1(2 \%)$ scored $1 / 5$ (much less comfortable). Twenty-eight $(55 \%)$ respondents thought that medical students should 
know more about the personal lives of the donors who they will dissect, $17(33 \%)$ were ambiguous to this idea, and 6 $(12 \%)$ felt that students should not know more about donors. When asked to score on a 5-point scale how helpful they felt watching a donor interview would be to medical students who wanted to know more about their donor, $30(58 \%)$ scored 5/5 (very helpful), $9(17 \%)$ scored 4/5, $11(21 \%)$ scored $3 / 5$ (neither helpful nor unhelpful), no respondents scored $2 / 5$, and $2(4 \%)$ scored $1 / 5$. When asked how helpful they thought filming an interview would be to donors and/or their family members, 21 (41\%) scored 5/5 (very helpful), 8 (16\%) scored 4/5, 17 (33\%) scored 3/5 (neither helpful nor unhelpful), no respondents scored $2 / 5$, and $5(10 \%)$ scored $1 /$ 5 (very unhelpful). Finally, $39(81 \%)$ respondents said they would be interested in conducting an interview that could accompany their remains to the University of Michigan, and $9(19 \%)$ said they would not.

Respondents were also asked to comment on any concerns or reservation they had concerning their donation, their personal feelings about conducting an interview, and any other comments they felt compelled to make. One-hundred and six comments in all were collected. Thirty of these were to report that they had no concerns or reservations concerning their donation, and no respondents commented on any concerns or reservations that they had. Many respondents used the opportunity to give personal details about their lives and reasons why they felt comfortable donating. Many more reported ambiguity toward what happens to them and their remains after death. Some respondents wrote that they opposed this program because it would create unnecessary stress on the donors, while others wrote that they strongly supported a donor interview program because it might foster compassion early in the education of future physicians. One respondent reported confusion over whether they would be interviewed by family members or by the medical school.

\section{DISCUSSION}

\section{Need for a More Personal Student-Donor Relationship}

Although students were not specifically asked in this study about their opinions regarding a more personal student-donor relationship, many students left comments at the end of the survey addressing this issue. One student wrote, "I was curious about my donor from the moment I met her. I think it is important for students to realize that our donors were people who gave us an amazing gift. There is no better way to understand that than to see who your donor was before they became our donor." Another student wrote, "I think this is a wonderful idea. I always wished I had known more about my donor besides what diseases she had, or the information I could glean from little details like the color of her fingernails. I made up a name [and a life] for her because I wanted to feel more connected." The results of this study contribute to the growing body of evidence suggesting that students desire a more personal relationship with the donor and lends further support to a program that replaces our current anonymized teaching model with one that allows for greater student-donor proximity. A donor interview project wherein students have access to videotaped footage of donors discussing their lives and reasons for donating would eliminate the anonymity of donors in the dissection laboratory and could provide for students the personal proximity to donors that many of them desire.

\section{Anticipated Effects of Interviews on Students}

Students' expressed desire to develop a closer student-donor relationship may stem from their need to view themselves as humane and caring human beings during the course of human dissection, especially after they have achieved clinical detachment from the donors. Knowing the donor personally might facilitate the students' balancing of their clinical detachment with greater empathy, and a donor interview program would be helpful to them by emphasizing the donor as a person. For example, one student wrote, "There were times in anatomy when I had to divorce myself from the fact that this was a person in order to surmount the emotional barrier to dissecting the body. I had to view the donor as a cadaver rather than someone who had a life, a family, friends, and so on. In that sense, dissection was a multifaceted act-it was simultaneously gruesome, necessary, and yet artful as well. I think watching the video would temporarily make this emotional barrier more difficult to surmount, but we'd all surmount it anyway because the donor knew that would be his/her fate and in fact wanted that we should learn from his/ her physical body." Another student wrote, "An interview would provide greater perspective as to why the patient donated and allow us to feel an even greater sense of understanding and pride in our dissections." Because these students may be more clinically detached, they may also be more comfortable in the dissection laboratory. This enables them to focus more contently on the art of dissection than it's emotionality, leading apparently to greater satisfaction with the course of dissection.

In contrast, some students may have a difficult time ever acquiring the clinical detachment that enables them to comfortably participate in dissection. These students might find the idea of a donor interview program counterproductive to their already overcompensated sense of empathy for the donor. One student wrote, "[A donor interview] would have made the dissection process much more personal and more difficult. Seeing someone speaking and interacting while alive and then seeing them as a cadaver would be extremely difficult. I honestly don't think I would have been able to emotionally handle it." This student clearly struggled to reconcile his/her emotional attachment to the donor's personhood with the physical reality of death, and they are afraid that knowing the donor as a living person and not simply a dead body would be emotionally paralyzing. Many other students who opposed donor interviews focused on the violence of dissection: "The act of dissection is inherently violent and requires some degree of detachment. It would be very hard for me to cut open and pull apart someone whom I had gotten to watch breathe and talk in a video. I would struggle to take to pieces someone who had told me about his life experiences and the love of his life and his family." Some students expressed guilt about having dissected: "Dissection might be that much more difficult by sort of knowing the person who's body we're about to destroy for the sake of our education." Whereas other students even expressed shame: "I am too ashamed to learn more about [the donors]." These reactions may arise from the students' difficulty achieving clinical detachment from the donor. In stark contrast to the contentment expressed by those students who might struggle to remain emotionally attached to the donor, these students 
seem to find the dissection laboratory a much less comforting place as they are more focused on the "shame" of their "violent" dissections. These students are apparently on a different path to finding balance between empathy and clinical detachment. A donor interview program might threaten to imbalance their empathy for the donor even more and so they unequivocally oppose it. It is important to recognize for the sake of these students that the donor interview is not a multifaceted tool for helping all students to achieve balance between empathy and clinical detachment; it is useful solely to those students who desire a closer bond to, or alternatively less detachment from, the donor.

Hafferty (1991) recognized that students' complex and inconsistent reactions to human dissection could be explained by differing views of the donor either as a "biological specimen" or as a body with a "former living-human status." Students predisposed to the latter view are thought to be more vulnerable to distress as they place higher value on empathy for the donor as a person, and so have greater difficulty with the act of dissection. The former position similarly makes it easier for students to dissect as they primarily view the donor as an object of study. The results of this study as well as those of Lamdin et al. (2012) support Hafferty's (1991) observations. Interestingly, a fair number of students in this study and a number of senior medical school faculty wondered aloud whether those students who objected most to watching a donor interview (i.e. those struggling with too much empathetic attachment rather than clinical detachment) might be the students who have the most to gain from such a program. After all, human dissection is only the first of many emotionally challenging situations facing medical students, as most will see their patients die and be with those patients' family and friends during their first year of clinical study. The gross anatomy course is therefore an opportune time to help students reflect and make sense of these experiences so that they may better handle similar stressors in future clinical practice (Horne et al., 1990; Lamdin et al., 2012).

\section{Anticipated Effects of Interviews on Donors}

The results show that a majority of donor respondents are supportive of a donor interview program and interested in participating in such a program if it existed. One donor even asked at the end of the survey, "Can I help to promote the program?" Furthermore, the reasons cited by donors for their interest in this program were highly varied. One donor reported, "When I was studying medicine at [name of] University, I dissected cadavers. It made me question my religious beliefs, but I answer to a higher calling. I am very comfortable with my decision. What answers could not be found in my life, maybe could be found in my death." Another donor similarly hoped that an interview would help "students and doctors understand my illness." Other donors were motivated simply by a desire to be remembered, as one donor wrote, "I would like to leave some record of myself behind. I really don't think anyone is interested in getting to know the 'real me'." Many reported just wanting to be helpful, "I am happy to be able to be a part of the on-going search for better lives. I pray the medical students learn a great deal from my donation."

Several donors reported feeling equivocal about filming an interview and these donors' comments often focused on themes of spirit-body separation and futility in worrying about what happens to one's body after death. Although many of these donors expressed ambivalence in both the effects an interview would have on the donor and their willingness to participate in such program, they seemed very willing and eager in their comments to begin sharing their life histories. For example, one donor wrote, "I have no feelings one way or the other. I have lived a full and adventurous life. Had great experiences, met many interesting people, worked from age 12 until age 74. Met 2 US presidents, joined the Lady Marines when they were reorganized in 1955 [...]" and went on in great detail about these and many other experiences. Many other donors wrote similar life synopses, and many more wrote short medical histories. Considering that donors left personal history information in the survey without prompting, it seems apparent that those who claim to be ambivalent about conducting an interview are in fact very open and willing to do so and should therefore be included in any future attempts to sign donors up for this program.

\section{More Empathy and Respect for Donors}

The results show that strong student support for implementing a donor interview program exists at UMMS. Many students justified their support by commenting on the enhanced empathy and respect for donor personhood that could result from donor interviews. One student wrote, "I would defnitely support this program [because] the anatomy lab can be a really impersonal space sometimes and it's easy to forget that we are dissecting real people who had real lives and left behind real people who love them and miss them." Although many students expressed their belief that a high degree of respect for donors exists in the anatomy laboratory and that disrespectful behavior toward donor bodies is no longer a problem, evidence to the contrary unfortunately exists. Four students commented in the survey that they felt a donor interview program would bring needed improvement in student treatment of donor bodies, with one student claiming, "[This program] will help increase the humanistic qualities of anatomy and avoid some of the egregious and insensitive comments and actions made towards the donors due to a lack of a personal connection." Interestingly, one of the donor respondents had extensive experience teaching anatomical dissection, and this donor wrote, "I have seen students play and joke about the cadavers. I believe if they understood who the people were and a little about them there would be much more respect and gratitude." Still another student wrote, "I do think we could use some reminders about who our donors were as a way to promote more respect for the bodies. As time has gone on I think students have dissected in not the most respectful fashion, and I think that having more reminders about our donors being real people would help with this." This student recognized an inverse relationship between time spent in the dissection laboratory and respect for donor bodies. As students move further into the course of dissection, clinical detachment tends more and more to replace empathy for the donor (Crow et al., 2012). This creates an environment in which disrespectful behavior is more likely to occur, and further underscores the importance of developing a way to help students maintain donor empathy throughout their course.

It is interesting to note that although no donors reported having any concerns or reservations about their gift, many donors reported feeling much more comfortable with the donation process if they knew their interview would accompany their remains to UMMS. It seems reasonable, to the extent 
logistically possible, that these donors should be assigned to students who agree to view their interview. This way donors who foresee better peace of mind coming from filming an interview will be respected by ensuring that their interview is viewed by those students assigned to their dissection. The opinions of those donors who object to this study will be respected by maintaining voluntary participation in this program.

\section{Effect of Interviews on Perceived Clinical Relevance of Anatomy}

Interestingly, many students recognized the possibility that watching the donor interviews before dissection might increase the clinical relevance of anatomy. One student wrote, "[Seeing my donor's interview] would have motivated me to spend more time learning and appreciating the clinical importance of anatomy." Several students specifically commented that a donor interview would help them to make clinical correlations between what they find on dissection and the patient's own account of their illnesses. For example, " $A n$ interview video would provide great background data for our patient making the dissection even more of a learning experience. Looking into the donors life would also make the experience more meaningful as all of our future patients will provide us with an in-depth history before we perform any kind of procedure." Several donors also considered this potential benefit to donor interviews, as one commented, "I think it would be good for some students because it would give them a better background on the body to be dissected and what it has been through." Another donor wrote, "Interviews could give insight about the condition of organs related to lifestyle." Yet another donor wrote, "I believe that medical students should have the benefits of what the donor's life has been and what their health and illness may have contributed to effects on the body." Bergman et al. (2008) showed that student anatomy test performance relies in large part on teaching anatomy in a clinical context. Therefore, any program that increases student appreciation for anatomy's clinical relevance is likely to improve student acquisition of important anatomical concepts. Madill and Latchford (2005) also showed that medical students' degree of involvement in dissection and their capacity to remain concentrated on the actual learning of anatomical information is linked to their relative success managing the psychological impact of dissection. If donor interviews were perceived as helpful to students in managing the emotional complexities of dissection, it is reasonable to presume they might also help those students to take a more active and focused role in learning human anatomy.

\section{A Too Personal Relationship?}

One concern shared by students and donors was whether or not watching an interview might create a too personal student-donor relationship, one that may even exceed the limitations of a professional doctor-patient relationship. One student wrote, "I believe there should be some separation of doctor-patient, just as surgeons should not operate on their family and friends." One of the donor respondents similarly commented, "Doctors rarely operate on people they know or love because of the connection of emotion. Seems awkward to have personal knowledge or morbid to me." Others viewed this concept entirely differently, as one donor wrote, "Med students won't be working with corpses in their careers.
They'll be working with people. Exposing them to the idea that humans lie at the core of their profession and not bodies is important. It could foster compassion early in their education." A student further added, "Medicine, when it comes down to it, is really about people. We learn a tremendous amount of anatomic and medical information from our cadavers that will carry with us throughout our entire careers. Why not learn about them as people instead of just bodies?" Underlying this disagreement is the relative perceived depth of the donor interview. It is likely that some donors will share more than others in their respective interviews, but this would be similar to what students and doctors encounter when taking patient histories. Some patients are quick to share even the most intimate of details with a medical student without hardly any prompting, whereas others are a sealed lid, barely willing to share anything other than their chief complaint. It seems likely, therefore, that donor interviews would closely approximate the reality of patient history taking in clinical training, and so might provide a very appropriate level of personal detail to a medical student. It may even be appropriate to structure the donor interview as a patient history, complete with a history of terminal illness, medical and surgical histories, and family and social histories. Such an interview is furthermore likely to advance student appreciation for the clinical relevance of anatomy and so increase student interest in the donor and the anatomical sciences generally.

\section{Study Limitations}

The weak response rates for both the student and donor surveys preclude us from drawing any conclusions about actual prevalence of student and donor opinions regarding a donor interview project. Having a low response rate also increases the likelihood of our results being biased by having only those students and donors with strong feelings about donor interviews respond to the surveys. Considering though that this study was not designed to be quantitative, but a qualitative assessment of existing student and donor opinions, the low response rates and risk of biased results were considered acceptable. Furthermore, keeping participation in a donor interview project voluntary for both students and donors means that even if many students and donors oppose this program, its implementation is still worthwhile for those who are in favor of it. As this study demonstrates, there is a plurality of students and donors who are interested in participating in this study, thereby providing rationale for moving forward with its implementation. At the same time it should be pointed out that the investigation was focused on the University of Michigan Medical School exclusively and it would be interesting to see results of similar projects from other medical schools.

This study is also limited by the inability to clarify for confused respondents the proposed program via anonymous survey. Although no students reported confusion regarding the proposed project, two donors appear to have been confused by the survey primer. One donor responded, "Do you mean I have my family interview me on my reasons for my decision, or do you mean the 'school' will interview me?" Another donor made the following comment, "My only family member is [name give] and she lives in [city, state]. She would not take time to do this for either the Universityor me. I feel this is a wonderful idea. I'm so sorry I can't 
belp." It seems this donor believed that participating in the donor interview program would necessitate family participation. The confusion may be due to a poorly worded primer in the donor survey. These donors were the only two who seemed to have been confused by this primer, and their responses did not change the qualitative results of the study nor the conclusions we have drawn.

\section{CONCLUSION}

Hopkins (2012) in a short reflection on anatomy learning explains, "Dissection is learning-learning anatomy and learning to face death. The experience is a flux between the explicit task of dissection, gaining knowledge of the physical body, and implicit emotional reflection, acknowledging the life of a person and the gift of her body to help others learn." A growing body of evidence suggests that many medical students may need a more personal relationship with the donor to better facilitate their development of emotional and humanistic professionalism. Promoting a closer student-donor relationship could benefit students by allowing them to feel like humane and caring human beings during the dissection course and helping them to maintain empathy in the course at a time when they have become too clinically detached. By implementing a program wherein interviews of donors are videotaped and later shown to students participating in their dissection, students would be permitted a closer relationship with their donor. This study shows that many students at UMMS are in favor of such a program, and foresee potential benefits such as improving students' ability to balance empathy and detachment, greater respect for the donor in the anatomy laboratory, and greater clinical relevance of dissection and the anatomical sciences in general. This study also reveals that many donors are interested in participating in a donor interview program. Reasons provided by donors are varied, but include increased empathy shown toward the bodies and potentially future patients of participating medical students, an opportunity to pass on one's life history, hope that an interview would enhance the medical students dissection experience, and hope that an interview would help students and doctors better understand their diseases. Many students and donors oppose the creation of a donor interview project, and their opinions will be respected by maintaining voluntary participation in future phases of this study. Students who choose not to view a donor interview or express emotional difficulty with dissection of their donors could be offered an opportunity to view a different donor's interview, and/or be invited to discuss with faculty their reasons for not wanting to view a donor interview. This would help faculty better understand reasons for differing student reactions to dissection and contribute to the development of an anatomy curriculum that meets the needs of all medical students.

Future IRB approved phases of this study will include the collection and production of video recordings from donors who volunteer to relate their life experiences and reasons for donating to medical students. These videos will be made available to medical students throughout their dissection course on an anonymous and confidential website. Their content will be protected by the same rules of confidentiality that govern student-patient relationships throughout medical school. Students will voluntarily choose to watch or not watch a donor's video. Students will then be offered an opportunity to talk about this viewing and they will answer a survey concerning their experience. End-of-year surveys will also be administered to the entire dissecting class in order to compare the attitudes of those students who chose to watch an interview with those who did not.

The donor interview program proposed would further advance our knowledge of donor and medical student attitudes toward human dissection and so could inform future decisions on the best way to conduct medical school anatomical dissection courses. Study results that support either of the hypotheses that donor interviews enhance the human dissection experience for students and/or the donation experience for donors would provide evidence in support of creating a more permanent donor interview program. Such a development in the fields of anatomical donation and anatomy education would be groundbreaking, and could positively affect both anatomical donation programs and anatomical science departments globally.

\section{ACKNOWLEDGMENTS}

The authors thank Ms. Carolyn Y. White for administrative assistance with this project and Kelli Sullivan Ph.D. for thoughtful comments.

\section{NOTES ON CONTRIBUTORS}

MICHAEL A. BOHL, B.A., is a fourth year medical student at the University of Michigan Medical School, Ann Arbor, Michigan. His research interests include medical education and the ethics of anatomical dissection.

ALEXIS HOLMAN, B.S., is a recent graduate of the University of Michigan, Ann Arbor, Michigan. She hopes to attend medical school, and her research interests include brain imaging and mood disorders.

DEAN A. MUELLER, M.O.L., has been the coordinator for the Anatomical Donations Program since 1995 at the University of Michigan Medical School, Ann Arbor, Michigan. His interests include leadership and ethics pertaining to anatomical donation.

LARRY D. GRUPPEN, Ph.D., is the Josiah Macy Jr. professor of medical education and Chair of the Department of Medical Education at the University of Michigan Medical School, Ann Arbor, Michigan. His research interests include faculty and leadership development and outcomes-based educational assessment.

SABINE HILDEBRANDT, M.D., is a lecturer in the Division of Anatomical Sciences, Department of Medical Education, at the University of Michigan Medical School Ann Arbor, Michigan. She teaches undergraduate and graduate courses of anatomy. Her research interest is in history and ethics of anatomy.

\section{LITERATURE CITED}

Bergman EM, Prince KJ, Drukker J, van der Vleuten CP, Scherpbier AJ. 2008. How much anatomy is enough? Anat Sci Educ 1:184-188.

Bertman SL, Marks SC Jr. 1985. Humanities in medical education: rationale and resources for the dissection laboratory. Med Educ 19:374-381.

Bohl M, Bosch P, Hildebrandt S. 2011. Medical students' perceptions of the body donor as a "first patient" or "teacher": A pilot study. Anat Sci Educ 4:208-213

Coulehan JL, Williams PC, Landis D, Naser C. 1995. The first patient: Reflections and stories about the anatomy cadaver. Teach Learn Med 7:61-66.

Crow SM, O'Donoghue D, Vannatta JB, Thompson BM. 2012. Meeting the 
family: Promoting humanism in gross anatomy. Teach Learn Med 24:49-54.

Druce M, Johnson MH. 1994. Human dissection and attitudes of preclinical students to death and bereavement. Clin Anat 7:42-49.

Hafferty FW. 1991. Into the Valley: Death and the Socialization of Medical Students. 1st Ed. New Haven, CT: Yale University Press. 288 p.

Hopkins R. 2012. Teaching and learning moments: A negotiation between death and learning. Acad Med 87:331.

Horne DJ, Tiller JW, Eizenberg N, Tashevska M, Biddle N. 1990. Reactions of first-year medical students to their initial encounter with a cadaver in the dissecting room. Acad Med 65:645-646.

Lamdin R, Weller J, Kerse N. 2012. Orientation to dissection: Assisting students through the transition. Clin Anat 25:235-240.

Madill A, Latchford G. 2005. Identity change and the human dissection experience over the first year of medical training. Soc Sci Med 60:1637-1647.

Penney JC. 1985. Reactions of medical students to dissection. J Med Educ 60:58-60.

Quilligan K. 2010. IUN teaches compassion, respect for patients.Nwi.com 29 August 2010. The Times Media Co, Munster, IN. URL: http://www.nwitimes. $\mathrm{com} /$ news/special-section/heath-innovation/article_08f2bea7-96dc-583f-a48359f8f21bbd76.html [accessed 20 July 2012].

Rizzolo LJ. 2002. Human dissection: An approach to interweaving the traditional and humanistic goals of medical education. Anat Rec 269:242-248.

Talarico EF Jr, Prather AD. 2007. A piece of my mind. Connecting the dots to make a difference. JAMA 298:381-382.

Trotman P (Director/producer). 2009. Donated to Science (DVD). Dunedin, New Zealand: Paul Trotman Films. 80 min.

Vannatta JB, Crow SM. 2007. Enhancing humanism through gross anatomy: A pre-course intervention. Med Educ 41:1108.

Wear D. 1989. Cadaver talk: Medical students' accounts of their year-long experience. Death Studies 13:379-391.

Weeks SE, Harris EE, Kinzey WG. 1995. Human gross anatomy: A crucial time to encourage respect and compassion in students. Clin Anat 8:69-79.

\section{APPENDIX A}

\section{Letter to Medical Students}

Dear Classmates,

You may remember receiving a survey last year on your thoughts and opinions about an approach to anatomical dissection taken by many Eastern medical schools wherein the donor is approached as a teacher rather than a "first patient." The response to the survey was fantastic (thank you to all who responded) and the results were very interesting. Eightyfour percent of you reported a preference for viewing the donor as a teacher, and $76 \%$ of you reported a desire to know more personal information about the donor. The results of this study were published, and Dr. Hildebrandt tells me that several of her colleagues here and abroad have taken a great deal of interest in what our next steps will be.

We are hoping to begin taking steps this summer toward instituting a new project in our Anatomical Donations Program wherein donors will be given an opportunity to conduct a video-recorded interview focusing on their lives and reasons for donating. These interviews will then be made available to medical students at the time of dissection. Participation of donors and medical students would, of course, be voluntary (and anonymous for students). Before starting this project, however, we want to know how you feel about it. I know how inundated we can all be with surveys, but your responses to this survey matter a great deal and will help shape the future of our anatomy program, and potentially medical schools nationwide.

\section{APPENDIX B}

\section{Text of Survey of Students for Donor-Interview Project, Phase I}

Should filmed interviews of donors be made available to medical students during the dissection course?

As University of Michigan medical students, you were surveyed last year on your attitudes and opinions toward the anatomical dissection course. You were asked specifically about our approach to the donor as a "first patient," and this was juxtaposed with how some Eastern medical schools approach donors as "great teachers." The results of this survey were published in an article titled Medical students' perceptions of the body donor as a "first patient" or "teacher": A pilot study (Bohl et al., 2011; Anat Sci Educ $4: 208-213)$. Interestingly, a majority of you felt that you wanted to know more personal information about the person who donated their remains for the sake of our medical educations, and that our current approach to dissection does not allow for an ideal donor-student relationship.

Some medical school have started providing medical students with more information about the donor, e.g. with videotapes of interviews given by donors and their families before death. Before the University of Michigan Donation Program starts on a similar interview project, we want to ask our students about their opinions and expectations.

We would like to know how you feel about instituting a program at the University of Michigan wherein donors are offered the opportunity to film an interview before death. This interview would be stored electronically along with the rest of the donor's information, and would be made available to students who are involved in the dissection of that donor. Student access to interviews would be voluntary and confidential, and the interviews could be watched prior, during, or after the dissection course.

The following survey is absolutely confidential and your answers are collected anonymously.

1. An interview of the donor you dissected were made available to you, would you watch it?
$\square$ No
$\square$ I don't know
$\square$ Yes

2. Do you think that watching an interview of your donor would have been beneficial to you during the anatomical dissection course?
$\square$ No
$\square$ I don't know
$\square$ Yes

3. Do you think watching an interview of your donor would have caused you undue distress?
$\square$ No
$\square$ I don't know
$\square$ Yes

4. Valuable would an interview of your donor be to you personally?
$\square$ (1) Not at all valuable
$\square(2)$
$\square$ (3) Moderately valuable
$\square(4)$
$\square$ (5) Extremely valuable 
5. Just as students are required to attend opening and closing ceremonies that honor the donors, do you feel that students should be required to watch a donor interview if available, in order to honor that person being dissected?

$\square$ No

$\square$ I don't know

$\square$ Yes

6. Provide in your own words reasons why you do or do not support the institution of a donor interview program at the University of Michigan.

7. Use the space below for any other thoughts, questions, or comments.

\section{APPENDIX C}

\section{Letter to Body Donors and Potential Donors}

Dear University of Michigan Donor/Potential Donor,

We in the Anatomical Donations Program are always looking for ways to improve upon the donation process so that we can be sure to make the absolute best use of your gift. We recently learned of a project at a different medical school where donors were interviewed about their lives and reasons for donating, and then videos of the interviews were shown to those medical students who benefited from that person's donation. We would like to know how you feel about such a program, and would be greatly appreciative if you would give us your insight by filling out a short survey. You can fill out the anonymous survey included here and send it back to us in the addressed and prepaid envelope.

OR

You can access and submit the survey online at the following web address:

URL: https://umichumhs.qualtrics.com/SE/?SID=SV_6Al2 z23CblIwL5y

Thank you so much for your time,

University of Michigan Donations Program

"If you have additional concerns, please complete the survey before calling us with questions regarding this project.

\section{APPENDIX D}

\section{Text of Survey of Body Donors for Donor-Interview Project, Phase I}

Should video recorded interviews of donors be given to students at the time of dissection?

Medical students at the University of Michigan were recently surveyed on their opinions of the human body dissection course. It was discovered through this survey that a majority of students would like to know more about the person who so graciously donated their remains for the sake of medical student education. Students may feel a need to have a better understanding of the person who made such a tremendous sacrifice on behalf of their own education in order to see them more fully as human beings throughout the dissection course. On the other hand, some other students might have a more difficult time completing a dissection if they feel they know the donor personally.
Similarly, it has been found that some donors and their family members greatly appreciate the opportunity to share personal stories with medical students and faculty, perhaps because this enables them to pass their personalities and life histories along with their remains. Some donors, however, may not want their personal information shared with medical students and faculty, or conducting an end-of-life interview may be too emotionally distressing.

We are currently exploring the option of interviewing potential donors and their families and making videotapes of these interviews available to medical students, as has been done at another medical school. However, before we begin such an interview project, we want to find out what our own donors and their families are thinking about such an idea.

We would like to know what you think of such a program. The following survey is absolutely confidential and your answers are collected anonymously.

1. Are you a donor or potential donor?

$\square$ Potential donor

$\square$ Donor

2. How open are you to filming an interview that will accompany your remains to the medical school?

$\square$ Not at all open/will absolutely not do an interview

$\square$ Neither open nor closed to the idea

$\square$ Very open/would love to do an interview

3. Do you have any worries or reservations concerning your gift? Please explain.

4. Would you feel more comfortable with the donation process if you knew that an interview depicting your life and your personality would accompany your remains while in the medical school?

$\square$ No

$\square$ Neither yes or no

$\square$ Yes

5. Would you be MORE or LESS comfortable with donating if you knew that those people handling your remains knew you more personally through a video interview?

$\square$ (1) Much LESS comfortable

$\square(2)$

$\square$ (3) Neither more nor less comfortable

$\square(4)$

$\square$ (5) Much MORE comfortable

6. Should medical students know more about the personal lives of those people who they will dissect in the course of their medical education?

$\square$ No

$\square$ Neither yes or no

$\square$ Yes

7. How helpful do you think watching a donor interview would be to medical students who want to know more about the donor?

$\square$ (1) Very helpful

$\square(2)$

$\square$ (3) Neither helpful nor unhelpful

$\square(4)$

$\square$ (5) Very helpful 
8. How helpful do you think filming an interview would be to donors and/or their family members?

$\square$ (1) Very helpful

$\square(2)$

$\square$ (3) Neither helpful nor unhelpful

$\square(4)$

$\square$ (5) Very helpful

9. In your own words, please explain how you personally feel about conducting interviews of donors before death.
10. Are you interested in conducting an interview that will accompany your remains, or those of your loved one, to the University of Michigan?

$$
\square \text { Yes }
$$

$\square$ No

Thank you very much for taking the time to answer this survey. Your opinions are very important to us, as is your comfort with the donation process. Respect for donors and their wishes is of the utmost importance to us and our students, and we are always striving to improve upon the donation process. Please use the space below to provide any comments you would like. 